\section{The physiology of cerebral blood flow during cardiopulmonary resuscitation}

Ivor D. Berkowitz MB B CH, Mark C. Rogers MD
For decades after Kouwenhoven's original description of cardiopulmonary resuscitation (CPR), ' antegrade blood flow during CPR was generally assumed to result from the direct compression of the heart between the sternum and the vertebral column, thereby forcing blood out of the ventricle - "a cardiac pump" mechanism of blood flow. If this hypothesis is correct, the following haemodynamic findings and flow events would be expected. During chest compression or "systole," a ventricular-atrial pressure gradient should develop that would close the atrioventricular valves. Synchronous left and right ventricular ejection would be associated with a reduction in ventricular volume. During chest relaxation or "diastole" ventricular pressure would fall below atrial pressure with subsequent atrioventricular valve opening and ventricular filling. Such a mechanism of blood flow generation is obviously operative during open-chest, internal cardiac massage.

Several observations of early investigators, however, did not support the cardiac pump hypothesis of antegrade blood flow. Weale and Rothwell-Jackson ${ }^{2}$ measured equivalent elevations in arterial and right atrial pressures with external chest compression and Gall ef al. using a cardioscope demonstrated that the mitral valve is open during chest compression. Other clinical observations also raised questions about direct cardiac compression as a sole mechanism of blood flow. CPR has certainly been successful in rcsuscitating either extremely large individuals or patients with severe emphysema. Nevertheless, in these patients it is physically impossible to depress the sternum enough to squeeze the heart against the stemum. External CPR is frequently ineffective in patients with flail chests, a situation that should more readily permit direct cardiac compression. ${ }^{3}$ Furthermore, a knowledge of anatomy would reveal that it is the right ventricle that is placed below the sternum and that the left ventricle is laterally placed and less likely to be squeezed by chest compression.

Despite these observations, it was not until the dramatic observations of Criley in 1976, ${ }^{4,5}$ that Kouwenhoven's cardiac compression hypothesis was seriously ques tioned. Criley reported that several patients who developed ventricular fibrillation during cardiac catheteriza- tion maintain a cardiac output adequate to maintain consciousness by repetitive coughing and phasically elevating intrathoracic pressure. This observation of "congh CPR" focused research on whether either direct cardiac compression or large fluctuations in intrathoracic pressure was the mechanism for forward blood flow during $\mathrm{CPR}$.

In 1980, the demonstration that manoeuvres that increased intrathoracic pressure with chest compression, increased aortic pressure and carotid blood flow during $\mathrm{CPR}$, led to a new theory accounting for forward blood flow during CPR. ${ }^{6.7}$ This "thoracic pump" hypothesis proposed that blood flow produced by external chest compression could be generated by phasic changes in intrathoracic pressure without the direct compression of the heart. According to this model, external chest compression produced a generalized elevation of intrathoracic pressure, that was transmitted equally to all cardiac chambers and intrathoracic vascular structures. This increase in intravascular pressure was transmitted from the intrathoracic to extrathoracic arteries but not to extrathoracic veins because of the presence of venous valves, a compliant extrathoracic venous system and easily collapsible veins but not arteries at the thoracic inlet. The difference in pressure between the intrathoracic arteries and extrathoracic veins caused blood to move from the chest into the extrathoracic vascular system. ${ }^{6,7}$

The "thoracic pump" mechanism implies that with chest compression-"systole" blood flows from the lungs through the left atrium, and an open mitral valve, left ventricle and aorta into the systemic circulation. During the release of chest compression-"diastole" intrathoracic pressure falls below that of the extrathoracic venous pressure and blood returns to the lungs. With this

Department of Anesthesiology/Critical Care Medicine,

The Johns Hopkins Medical Institutions, Baltimore, Maryland. Address correspondence ra: Dr. Ivor D. Bcrkowitz, Department of Anesthesiology/Critical Care Medicine, The Johns Hopkins Hospital, 600 North Wolfe Street - Halsted 842, Baltimore, Maryland 21205. 
mechanism, the heart plays no active role as a blood pump, but is only a passive conduit. ${ }^{\text {? }}$

Much cxperimental data has acsumulated over the past several years that supports the thoracic pump mechanism of antegrade blood flow during CPR. Two dimensional echocardiographic studies during CPR in humans showed that during chest compression the aortic and milral valve are open and that left ventricular dimensions are not reduced. ${ }^{8}$ Angiographic studies with contrast injection in the pulnonary vein have confirmed that blood flows through the left atrium, and an open mitral valve to the left ventricle and aorta during a single chest compression, and from the vena cava to the lung during diastole. ${ }^{9.10}$ These are findings that would be predicted by the thoracic pump mechanism in which the heart is purely a conduit and not responsible for pumping blood.

Further support for a "thoracic pump" mechanism has emerged from studies that have evaluated the haemodynamic response to varying compression rates and duty cycles during CPR. "1 If blood flow is generated by direct cardiac compression and the stroke volume is determined by the compression force and the amount of deformation of the heart, then prolonging compression or duty cycle beyond the time necessary to completely squeeze blood from the heart should have no further effect on stroke volume. Increasing the compression rate will increasc flow, since a fixed volume is ejected per unit time. If blood flow is by the "thoracic pump" then the time required to eject blood from the large capacitance lung vasculature is prolonged in comparison with a cardiac pump mechanism. Flow will be enhanced by both prolonging the duty cycle and increasing compression force, but will be insensitive to increasing rate. Halperin er al. ${ }^{11}$ studied the haemodynamic response to changes in rate and duty-cycle during manual CPR in dogs, and compared the cerebral (CBF) and myocardial blood flows and their respective perfusion pressures with those obtained during resuscitation with vest CPR (a CPR technique where flow is produced only by the thracic pump mechanism) and in a group in which only direct cardiac compression was produced. In this group of animals, either the sternum was removed and the piston of the pneumatic compressor device (Thumper) was placed directly on the exposed heart, or open chest internal cardiac massage was performed. In the vest-CPR group, increased perfusion pressures and flows were produced by increasing the duty-cycle and not by changing compression rate whereas in the direct cardiac compression group. increasing the rate but not the duty-cycle enhanced both pressures and flows. They also demonstrated that the haemodynamic response to closed chest cardjac massage mirrored that of the vest CPR group. Perfusion pressures and flows were responsive to changes in duty-cycle and not sensitive to rate changes, providing further evidence that antegrade blood flow during closed chest massage in large dogs is produced by the thoracic pump mechanism.

The intrathoracic pump mechanism of antegrade blood flow is, however, not universally accepted. Whereas the thoracic pump may be operative in large dogs with keel-shaped chests that limit cardiac compression, direct cardiac compression may play a role during CPR in smaller dogs with flatter chests ${ }^{12}$ and in neonates and infants ${ }^{13}$ with compliant and readily compressible chest walls. Some investigators have suggested that the discrepant findings concerning the mechanism of blood flow might relect differences in compression techniques, ${ }^{14}$

Maier et al. ${ }^{15}$ studied left ventricular dynamics with ultrasonic transducers to measure ventricular dimensions during CPR in chronically instrumented dogs. They demonstrated that high impulse CPR (brief, high velocity chest compressions) produced changes in left ventricular shape that were characterized by shortening of the axis parallel to the force vector and elongation of the other two axis. In addition, peak cardiac and vascular pressures were consistently two to four times greater than the pleural pressure. The large differences between intracardiac and pleural pressures and the changes in the ventricular shape during chest compression implied that direct cardiac compression was a major determinant of stroke volume. However, although these investigators demonstrated that changes in ventricular shape occurred with chest compressions, concommitant changes in chamber volume that are responsible for the stroke volume in a cardiac pump, were not investigated. In another study, Feneley ${ }^{14}$ demonstrated that high impulse, manual chest compressions produced mitral valve closure, no anterograde mitral valve flow together with left ventricular deformation - findings supportive of the cardiac pump mechanism.

\section{Mechanical methods to improve CPR}

If phasic elevations of intrathoracic pressure are responsible for antegrade blood flow during CPR, then for the same degree of stemal displacement, manoeuvres that enhance these pressure fluctuations should enhance cardiac output during CPR. Various mechanical techniques that increase intrathoracic pressure during CPR have been investigated in both laboratory and clinical situations.

Simultaneous chest compression and ventilation at high airway pressures (SCV-CPR) is a logical therapeutic extension of the above-described pathophysiology. By sinultaneously compressing the chest while providing ventilation at high airway pressures $(80-100 \mathrm{mmHg})$ it is possible to enhance both intrathoracic pressure during chest compression and cardiac output compared with 
conventional CPR. Chandra et al. first demonstrated that SCV-CPR results in dramatic increase in blood pressure and carotid blood flow when compared with conventional CPR in animals ${ }^{16}$ and humans. ${ }^{17}$ Subsequent studies have confirmed the efficacy of this technique in enhancing cerebral perfusion in large animal models of $\mathrm{CPR}^{18,19}$ SCV-CPR does not, however, enhance intrathoracic pressure and blood flow in small dogs ${ }^{12.20}$ and piglets, ${ }^{21}$ animals with relatively flattened and compliant chest when compared with conventional CPR (CON-CPR). In these animals, perhaps because of the differences in chest geometry when compared with large animals, high levels of intrathoracic pressure could be generated by CON-CPR alone. ${ }^{22}$ Indeed, the intrathoracic pressure exceeded the peak airway pressure achieved during SCV-CPR, If antegrade flow in these models is produced by the thoracic pump mechanism, then utilizing lower peak airway pressures for SCV.CPR than the high intrathoracic pressure that could be gencrated by CON-CPR would not be expected to enhance intrathoracic pressure and blood flow. Direct compression of the heart may also contribute to flow in these small animals.

Abdominal binding during CPR in humans ${ }^{23}$ and $\operatorname{dog} s^{3.18,24}$ increases both aortic blood pressure and carotid blood flow. Higher intrathoracic pressures are produced by limiting the caudal movement of the diaphragm during chest compression. The bound abdominal cavity becomes a second compartment in continuity with the thoracic pump. ${ }^{25}$ Other mechanisms that contribute to the improved flow with this technique include the redirection of blood flow from organs below the diaphragm, to increase the central circulatory blood volume, and possibly lessen carotid collapse.

CPR performed by the phasic inflation at high pressure of a circumferential vest placed around the thorax-vest CPR has been investigated in the laboratory as a CPR technique without the need for complex simultaneous ventilation devices. ${ }^{26}$ Halperin et al. have in addition recently initiated a clinical trial of this CPR method at the Johns Hopkins Hospital. In evaluating this technique, the advantages of vest inflation over conventional CPR were considered twofold. ${ }^{26}$ The volume of the thorax would be more efficiently reduced and the intrathoracic pressure subsequently increased by a uniform, small decrcase in chest dimensions than by the distortion of a small area from a relatively large sternal displacement. ${ }^{26}$ The chest wall trauma and visceral injury not infrequently associated with conventional CPR might also be avoided. in dogs, vest $C P R$ at very high vest pressures $(>300 \mathrm{mmHg}$ ) generated essentially normal myocardial and cerebral blood flows, but also produced severe myocardial and pulmonary contusions. At lower pressures (280 $\mathrm{mmHg}$ ) no visceral trauma was produced. CBF was maintained at control values while myocardial fows were reduced by 50 per cent.

Interposed abdominal counterpulsation CPR (IAC. CPR) may be considered as an example of a third mechanism for the generation of antegrade blood flow during CPR, the "abdominal pump."27 Abdominal compressions are applied during the relaxation phase of chest compression. The action of this abdominal pump resembles that of an intraaortic balloon pump. Abdominal counterpulsation increases venous return and also compresses the abdominal aorta to produce retrograde aortic flow that closes the aortic valve and increases central aortic diastolic pressure. ${ }^{28,29}$ An increase in the effective duty-cycle may also be operative since the abdominal compression partially sustains the increase in intrathoracic pressure of the chest compression phase. ${ }^{25}$ Cardiac output, systemic oxygen uptake, myocardial and cerebral blood flow are substantially enhanced with this technique compared with CON-CPR. ${ }^{28-30}$

The assumption that carotid blood flow reflects cerebral flow and that manoeuvres that increase carotid flow will increase cerebral blood flow is implied in the many studies noted above. However, this is nor necessarily valid, since carotid blood flow is not cerebral blood flow. The carotid artery supplies blood not only to the brain by the internal carotid artery but also, and particularly in primates, to extracranial cephalic tissue such as the tongue, scalp and muscles of the head and neck, via the external carotid branch. ${ }^{25}$ In acdition, the vertebral and spinal arteries provide a varying component of cerebral blood flow. This phenomenon is well known to intensivists in the setting of clinical brain death. Such patients may have bounding carotid pulses and well-perfused faces and scalps and yet have no documented cerebral blood flow.

\section{Intracranial pressure and cerebral bloud now during CPR}

The cerebral circulation during normal physiology and during CPR is considered an example of a vascular waterfall in which the carotid artery pressure and either the intracranial (ICP) or jugular venous pressures are regarded as the effective upstream and downstream pressures respectively. ${ }^{25}$ The cerebral perfusion pressure will therefore be reflected by this pressure gradient. Much of the previous discussion has centred around techniques that enhance intrathoracic pressure and hence aortic and carotid pressure, but they do, in addition, have the potential for independently elevating ICP. To the cxtent that intrathoracic pressure is in some way transmitted to the intracranial vault, a dilemma is created. ${ }^{31.32}$ Enhancing intrathoracic pressure and thereby aortic and carotid pressure would also variably elevate ICP, the downstream 


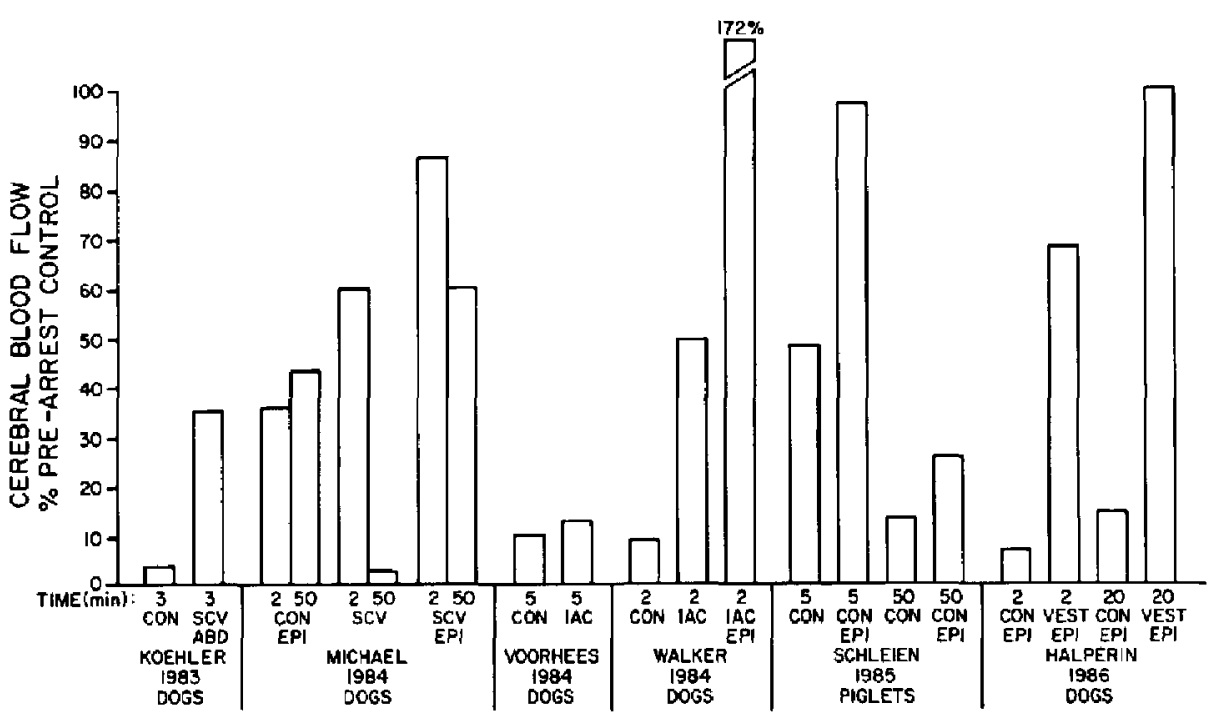

FIGURE Cerebral blood flow generated by various CPR techniques. Time denotes duration of CPR; CON indicates conventional CPR; $5 C V$, simultaneous compression and ventilation CPR; IAC, interposed abdominal compression CPR; ABD, abdominal binding, and EPI, epinephrine used. Adapted from: Geller SA. Elliou PL, Rogers MD. Update on cardiopulmonary resuscitation. In: Advances in Anesihesin and Critical Care $1986 ; 3: 323-48$.

pressure, and potentially fail to improve cerebral perfusion. Thus, it is not always clear whether strategies that will increase intrathoracic pressure will necessarily improve cerebral perfusion. ${ }^{25}$

The fact that elevations in pleural pressure can increase ICP has been known for the past decade. While this has usually been considered in the setting of head-injured patients with cerebral oedema who require ventilatory support with PEEP, a similar interaction between increased intrathoracic pressures and elevated ICP occurs during CPR. That this is of more than theorctical intercst became evident when Rogers et al. ${ }^{32}$ described patients who sustained a cardiac arrest in an intensive care unit while ICP was being monitored with a subarachnoid bolt. CPR generated an adequate blood pressure but also large phasic elevations in ICP, such that cerebral perfusion pressure was minimal in spite of adequate CPR by traditional standards. Such large pulsatile increases in ICP with chest compressions have been repeatedly demonstrated in laboratory studies. ${ }^{18,33}$

The transmission of intrathoracic pressure to the intracranial compartment can theoretically occur through either the arterial or venous systems or via the CSF through thoracic vertebral foramina. Guerci et al. ${ }^{31}$ evaluated the contribution of each of these potential pathways in a dog model of CPR. The valved jugular venous system and carotid arterial system did not contribute to the elevated ICP. Transmission of intrathoracic pressure occurred via the valveless, non-jugular veins such as the vertebral venous plexus, as well as via the thoracic CSF, most likely via the intervertebral foramina.

Another cerebral flow limiting factor that has been demonstrated in animal CPR studies is arterial collapse. ${ }^{33}$ This occurs at the thoracic inlet when the pressure surrounding the intrathoracic carotid artery exceeds its intraluminal pressure and the vessel narrows, impairing pressure transmission ot the extrathoracic arterial system. Alpha agonists both increase the intrinsic resistance of the carotid artery to collapse and by producing intense vasoconstriction reduce the peripheral runoff that enhances callapse. ${ }^{33}$

The Figure displays the results of cerebral blood flow measurements made during CPR in various animal models utilizing various new mechanical techniques. In order to place the flows obtained during these studies in clinical perspective comparison will be made with CBF measurements and neurologic events from other clinical settings and laboratory investigations. Studies of patients undergoing carotid endarterectomy have shown that CBF of $15-20 \mathrm{ml} \cdot 100 \mathrm{gm}^{-1} \cdot \mathrm{min}^{-1}$, approximately $30 \mathrm{per}$ 
cent of normal flow, is the threshold below which adverse effects develop. ${ }^{34}$ During cerebral ischuemia, EEG amplitude decreases with flows as high as $30 \mathrm{ml} \cdot 100$ $\mathrm{gm}^{-1} \cdot \mathrm{min}^{-1}$ and in man flattens if flow falls below 16 $\mathrm{ml} \cdot 100 \mathrm{gm}^{-1} \cdot \mathrm{min}^{-1}{ }^{34}$ In baboons, suppression of evoked cortical potentials is noted with flows of 15-20 $\mathrm{ml} \cdot 100 \mathrm{gm}^{-1} \cdot \min ^{-1}{ }^{35}$ A blood flow of $8-10 \mathrm{ml} \cdot 100$ $\mathrm{gm}^{-1} \cdot \mathrm{min}^{-1}$ results in an intracellular discharge of potassium, an event indicative of cell pump failure.

\section{Pharmacologic manipulation of cerebral blood flow during CPR}

Several experimental studies performed over the past 20 years have demonstrated that alpha adrenergic agonists improve the outcome of CPR. ${ }^{37,38}$ These drugs cause vasoconstriction of peripheral arterioles and increasc aortic diastolic pressure and myocardial perfusion. Until recently, however, the effect of these catecholamines on CBF during CPR was unknown. Early studies of SCVCPR demonstrated dramatic increases in carotid blood flow. Adrenergic drugs, by virtue of their extracerebral vasoconstrictive properties, might divert the enhanced flow from the extracerebral to the cerebral vascular beds. Michael et al. showed that epinephrine administration during CPR, by its vasconstrictive properties, dramatically reduced the blood flow in the well-perfused extracranial cephalic tissues such as tongue, facial skin and muscle, redirecting this flow into the cerebral circulation. ${ }^{33}$ With continuous epinephrine infusion, blood flow of approximately 70 per cent of baseline could be maintained for $60 \mathrm{~min}$ of SCV-CPR. Without epinephrine, cerebral perfusion was only one per cent of prearrest values (Figure). Epinephrine also dramatically increased CBF during conventional CPR. ${ }^{13,33}$ Epinephrine and other alpha agonists, in addition to redistributing blood flow to the cerebral circulation, also prevent carotid collapse, thereby maintaining the upstream pressure for cerebral perfusion. ${ }^{33}$

Whether pure alpha agonists or catecholamines with both alpha and beta properties are more effective in enhanicing CBF during CPR is currently being investigated. ${ }^{39-41}$ With an intact blood-brain barrier, circulating catecholamines have little effect on CBF and cerebral metabolism. Distuption of this barrier may, however, develop during the cerebral ischaemia associated with cardiac arrest and resuscitation. ${ }^{25,42}$ Drugs with both alpha and beta action might be inferior to pure alpha agonists, since beta agonism might enhance cerebral metabolism and oxygen consumption in the face of limited CBF and oxygen delivery, aggravating ischaemia. ${ }^{43}$ However, studies in dogs comparing CBF, cerebral consumption of oxygen and somatosensory-evoked potentials during CPR commenced immediately after inducing ventricular fibrilla- tion and supported with either epinephrine or phenylephrine, revealed no difference. ${ }^{41}$

Whereas the use of agents with alpha agonist activity to increase resistance in extracerebral vessels selectively and thereby redirect blood flow to the cerebral circulation is effective, the selective vasodilation of the cerebral circulation to enhance CBF during CPR is less well studied. Nevertheless, much attention has been directed towards the use of calcium channel blockers in this regard. ${ }^{44}$ When the circulation is restored after global cerebral ischaemia that accompanies cardiac artest, a period of cerebral hyperemia is initially observed that is usually followed by prolonged hypoperfusion - the "no reflow" phenomenon. This hypoperfusion is caused by cerebral vasoconstriction that has been attributed to the influx of calcium into the smooth muscle cells of the ischaemic cerebral vasculature. ${ }^{44}$ If this hypothesis is correct, then the use of calcium channel blocking agents could potentially reduce cerebral vasoconstriction and enhance CBF.

Several calcium channel blockers have been evaluated to determine whether they can increase CBF during this "no reflow" phase following global cerebral ischaemia. The results have, however, been conflicting. Nimudipine appears to be the most selective cerebral vasodilator ${ }^{45}$ and in some studies increases $\mathrm{CBF}$ without increasing cerebral consumption of oxygen. ${ }^{46}$ Steen et al.$^{47}$ demonstrated in dogs that when nimodipine was administered prior to ischaemia, CBF almost doubled during the postischaemic hypoperfusion period and neurological recovery was improved when compared with the control group. When the drug was given after complete ischaemia, postischaemic flow improved but there was no improvement in neurologic recovery. ${ }^{48}$ In the monkey, improved CBF and neurologic outcome occurred with the administration of nimodipine during the postischaemic period..$^{49}$ White et al. ${ }^{50}$ demonstrated that in dogs pretreated with flunarizine 20 minutes prior to cardiac arrest, CBF was restored by reperfusion with cardiopulmonary bypass, compared with an untreated group that demonstrated no reflow. Deshpande et at. ${ }^{51}$ confirmed the protective effect of flunarizine in a rat model of cerebral ischaemia. Newberg, ${ }^{52}$ however, failed to demonstrate improvement in CBF and neurologic outcome by administering flunarizine after producing cerebral ischaemia in dogs. There have been conflicting results in the studies that have evaluated the effects of lidoflazine on CBF. White et al. ${ }^{53}$ reported that lidoflazine administered in the postischaenic period improved CBF and neurologic outcome. Dean et al. ${ }^{94}$ however, failed to confirm these beneficial results. Further studies are ongoing to better evaluate this class of drugs in improving postischaemic CBF and metabolism.

After remaining dormant for two decades, the field of CPR research has undergone a rebirth. Potential mecha- 
nisms of blood flow generation have been elucidated. New mechanical techniques that have enhanced blood flow have been developed and evaluated in the laboratory await clinical trials. Advances in pharmacologic techniques of brain resuscitation are appearing on the horizon and promise an exciting future for CPR research.

\section{References}

I Kouwenhoven WB, Jude JR, Knickerbocker GG. Closed chest cardiac massage. JAMA 1960; 173: 1064-7.

2 Weale FE, Rothwell-Jackson $R L$. The efficiency of cardiac massage. Lancet 1962; 1: 990-2.

3 Rudikoff MT, Maughan WL, Effron M, Freund P, Waisfeldt $M L$. Mechanisms of blood flow during cardiopulmonary resuscitation. Circulation 1980; 61: 345-52.

4 Criley JM, Blatufuss AH, Kissel GL. Cough-induced cardiac compression. JAMA 1976; 236: 1246-8

5 Rosborough JP, Hausknecht $M$, Niemann JT, Criley JM. Cough supported circulation. Crit Care Med 1981; 9: $371-2$.

6 Rudikoff MT, Maughan WL, Effron $M$ et al. Mechanisms of blood flow during cardiopulmonary resuscitation. Circulation 1980; 61: 345-52.

7 Weisfeldt $M L$, Chamdra N. Physiclogy of cardiopulmonary resuscitation. Ann Rev Med 1981; 32: 435-42.

8 Werner JA, Greene HL, Janko CL, Cobb LA. Visualization of cardiac valve motion in man during external chest compression using lwo-dimensiunal echocardiography.

Circulation 1981; 63: 1417-21.

9 Niemann JT, Rosborough JP, Hausknechi M, Garner D, Criley JM. Pressure-synchronized cincangiography during experimental cardiopulmonary resuscitation. Cirçulation 1981; 64: 985-91.

10 Cohen JM, Chandra N, Alderson PO, Aswegen AV, Tsitlik .IE, Weisfelde $M L$. Timing of pulmonary and systemic blood flow during intermittent high intrathoracic pressure cardiopulmonary resuscitation in the dog. Am J Cardiol $1981 ; 49: 1883-9$.

11 Halperin HR, Tsitlik JE, Guerci AD, et al. Determinants of blood flow to vital organs during cardiopulmonary resuscitation in dogs. Circulation 1986; 73: 539-50.

12 Babbs CF, Tacker WA, Paris RL, Murphy RJ, Davis RW. $C P R$ with simultaneous compression and ventilation at high airway pressure in 4 animal models. Crit Care Med $1982 ; 10: 501-4$.

13 Schteien CL, Dean.JM, Koehter RC et al. Effect of epinephrine on cerebral and myocardial perfusion in an infant animal preparation of cardiopulmonary resuscitation. Circulation 1986: 73: 809.

14 Feneley MP, Maier GW. Gaynor W et al. Sequence of mitral valve motion and transmitral blood flow during manual cardiopulmonary resuscitation in dogs. Circulation $1987 ; 76: 363-75$.
15 Maier GW, Tywon GS, Olven CO el al. The physiology of external cardiac massage: high-impulse cardiopulnonary resuscitation. Circulation 1984; 70: 86-101.

16 ChandraN, Weisfeldt ML, TsitlikJ et al. Augmentation of carotid flow during cardiopulmonary resuscitation by ventilation at high airway pressure simultancous with chest compression. Am J Cardiol 1981; 48: 1053-63.

17 Chandra $N$, Rudikoff $M$, Weisfeldt $M L$. Simultaneous chest compression and ventilation at high airway pressurc during cardiopulmonary resuscitation. Lancet 1980: 1: 175-8.

18 Kochler RC, Chandra N, Guerci AD et al. Augmentation of cerebral perfusion by simultaneous chest compres. sion and lung inflation with abdominal binding after cardiac arrest in dogs. Circulation 1983; 67: 266-75.

19 Luce $J M$, Ross $B K, O^{\prime} Q_{u}$ in $R J$ et al. Regional blood flow during cardiopulmonary resuscitation in dogs using simultaneous and nonsimultaneous compression and ventilation. Circulation 1983; 67: 258-64.

20 Sanders $A B$, Ewy GA, Alferness $C A, T a f t T$, Zimmerman $M$. Failure of one method of simultaneous chest compression, ventilation, and abdominal binding during CPR Cril Care Med 1982; 10: 509-13.

21 Berkowiz ID. Chamarajanisiri T, Koehler RC et al. Simultaneous compression and ventilation cardiopulmonary resuscitation does nor enhance cerebral and myocardial perfusion in an infant model of cardiopulmonary resuscitation. Anesthesiology 1986; 65: A 108.

22 Dean JM, Koehler RC, Schleien $C L$ et al. Age related changes in chest geometry during cardiopulmonary resuscitation. J Appl Physiol 1987; 62: 2212-19.

23 Chandra N, Snyder LD, Weisfeldt ML. Abdominal binding during cardiopulmonary resuscitation in man. JAMA $1981 ; 246: 351-3$.

24 Niemann JT, Rosborough JP. Ung S, Criley JM. Hemo dynamic effects of continuous abdominal binding during cardiac arrest and resuscitation. Am J Cardjol 1984; 53: 269-74.

25 Koehler RC, Michael JR. Cardiopulmonary resuscitation, brain blood flow, and neurologic recovery. Crit Care Clinies 1985; 1: 205-22.

26 Halperin $H R$, Guerci $A D$, Chandra $N$ et al. Vest inflation without simultaneous ventilation during cardiac arrest in dogs: improved survival from prolonged cardiopulmonary resuscitation. Circulation 1986; 74: 1407-15.

27 Babbs CF. Hemodynamic mechanisms in CPR: a theoretical rationale for resuscitative thoracotomy in non-traumatic cardiac anrest. Resuscitation 1987; 15: 37-50.

28 Voorhees WD, Babbs CF, Niebauer MJ. Improved oxygen delivery during cardiopulmonary resuscitation with interposed abdominal compressions. Ann Emerg Med 1983; 12: 128-35.

29 Voorhees WD, Ralston SH, Babbs CF. Rcgional blood 
fow during cardiopulmonary resuscitation with abdominal counterpulsation in dogs. Am J Emerg Med 1983; 2 : 123-8.

30 Walker IW, Bruestle JC, White BC, Evans AT, Indreri $R$, Bialek $H$. Perfusion of the cerebral cortex by use of abdominal counterpulsation during cardiopulmonary resuscitation. Am J Emerg Med 1984; 2: 391-3.

31 Guerci $A D$, Shi $A Y$, Levin $H, T$ sitik J, Weisfeldt $M L$. Chandra N. Transmission of intrathoracie pressure to the intracranial space during cardiopulmonary resuscitation in dogs. Circ Res 1985; 56: 20-30.

32 Regers MC, Nugent $S K$, Stidham GL. Effects of closedchest cardiac massage on intracianial pressure. Crit Care Med 1979; 7: 454-6.

33 Michael JR, Guerci AD, Koehler RC et al. Mechanisms by which epinephrine augments cerebral and myocardial perfusion during cardiopulmonary resuscitation in dogs. Circulation 1984; 69: 822-35.

34 Sundt TM, Sharbrough PW, Anderston RE et al. Cerebral blood flow measurements and electroencephalograms during sarotid endarterectomy. J Neurosurg 1974; $41 ; 310$ 20.

35 Branston NM, Symon L, Crockard HA et al. Relationship between the cortical evoked potential and local cortical blood flow following actec middle cerebral ancry occlusion in the baboon. Exp Neurol 1974; 45: 195-208.

36 Branston NM, Strong AJ, Symon L. Extracellular potassium activity, evoked potential and tissue blood flow: relationship during progressive ischemia in baboon cerebral cortex. J Neurol Sci 1977; 32: 305-21.

37 Pearson JW, Redding JS. Influence of peripheral vascular tone on cardiac resuscitation. Anesth Analg 1965; 44: 746.

38 Yakaitis $R W$, Otto $C$, Blit $C D$. Relative importanec of alpha and beta adrenergic receptors during resuscitation. Crit Care Med 1979; 7: 293.

39 Brown CG, Werman HA, Davis EA, Katz S, Hamiin RL The effect of high-dose phenylephrine versus epinephrine on regional cerebral blood flow during CPR. Ann Emerg Med 1987; 16: 743

40 Brown CG, Davis EA, Werman HA, Hamlin RL. Methoxamine versus epinephrine on regional cerebral blood flow during cardiopulmonary resuscitation. Crit Care Med 1987; 15: 682

41 Schleien $C L$, Gervais $H$, Berkowitz ID et al. Comparison of phenylephrine and epinephrine on cerebral and myocardial perfusion and somatosensory evoked potentials during and following cardiopulmonary resuscitation. Anesthesiology 1987; 67: A650.

42 Arai $T$. Watanabe $T$, Nagaro $T$ et al. Blood-brain barrier impairment after cardiac resuscitation. Crit Care Med 1981; 9: 444-8.
43 Carlsson $C$, Hagerdal $M, K a a s i k ~ A E$, Siesjo BK. A catecholamine-mediated incrcase in cerebral oxygen uptake during immobilization stress in rats. Brain Res 1977; 119 : 223.

44 Geller SA, Elliot PL, Rogers MC. Update on cardiopulmonary resuscitation. Adv Anesth and Crit Care 1986; 3: 323-48.

45 Kazda S, Tawart $R$. Nimodipine: a new calcium antagonistic drug with a preferential cerebrovascular action. Acta Neurochir 1982; 63: 259-65.

46 Mohamed AA, Mendelow AD, Teasdale GM, et al. Effect of the calcium antagonist nimodipine on local ccrcbral blood flow and metabolic coupling. J Cereb Blood Flow Metab 1985; 5: 26-33.

47 Steen PA, Newberg LA. Milde JH, and Michenfelder JD. Nimodipine improves cerebral blood flow and neurologic recovery after complete cerebral ischemia in the dog. J Cereb Blood Flow Metab 1983; 3: 38-43.

48 Steen PA, Newberg LA, Milde JH, Michenfelder JD. Cerebral blood flow and neurologic outcome when nimodipine is given after complete cerebral ischemia in the dog. J Cereb Blood Flow Metab 1984; 4: 82-7.

49 Steen PA, Gisvold SE, Milde JH, et al. Nimodipine improves outcome when given after complete cerebral ischemia in primates. Anesthesiology 1985; 62: 406-14.

50 White BC, Gadinski DS, Hoehner PJ et al. Effect of flunarizine on canine carebral cortical blood flow and vascular resistance post cardiac arrest. Ann Emerg Med 1982; 111: 119-26.

51 Deshpande $J K$, Wieloch $T$. Flunarizine, a calcium entry blocker, ameliorates ischemic brain damage in the rat. Anesthesiology 1986; 64: 215-24.

52 Newberg LA, Steen PA, Milde JH ef al. Failure of Hunarizine to improve ccrebral blood flow or neurologic recovery in a canine model of complete cerebral ischemia. Stroke 1984; 15: 666-71.

53 White BC, Winegar C. Hoehner $P$ et al. Use of calcium blockers in cerebral resuscitation, abstracted. J Trauma 1982; $22: 645$.

54 Dean $J M$, Hoehner PJ, Rogers $M C$ et al. Effect of lidoflazine on cerebral blood flow following 12 minutes total cerebral ischemia. Stroke 1984; 15: 531-5. 